\title{
Pirfenidone inhibits post-traumatic proliferative vitreoretinopathy
}

BNMK Khanum ${ }^{1,4}$, R Guha ${ }^{2,4}$, VP Sur ${ }^{2}$, S Nandi ${ }^{2}$, SK Basak ${ }^{3}$, A Konar $^{2}$ and S Hazra ${ }^{1}$

\begin{abstract}
Purpose The purpose of the study was to evaluate the efficacy and safety of intravitreal pirfenidone for inhibition of proliferative vitreoretinopathy (PVR) in a model of penetrating ocular injury.

Patients and methods Penetrating trauma was induced on the retina of rabbit and treated either with $0.1 \mathrm{ml}$ of phosphatebuffered saline (PBS) or $0.1 \mathrm{ml}$ of $0.5 \%$ pirfenidone, and development of PVR was evaluated clinically and graded after 1 month. Histopathology and immunohistochemistry with transforming growth factor beta (TGF $\beta$ ), alpha smooth muscle actin ( $\alpha$ SMA), and collagen- 1 were performed to assess the fibrotic changes. Expression of cytokines in the vitro-retinal tissues at different time points following pirfenidone and PBS injection was examined by RT-PCR.
\end{abstract} Availability of pirfenidone in the vitreous of rabbit at various time points was determined by high-performance liquid chromatography following injection of $0.1 \mathrm{ml}$ of $0.5 \%$ pirfenidone. In normal rabbit eye, $0.1 \mathrm{ml}$ of $0.5 \%$ pirfenidone was injected to evaluate any toxic effect.

Results Clinical assessment and grading revealed prevention of PVR formation in pirfenidone-treated animals, gross histology, and histopathology confirmed the observation. Immunohistochemistry showed prevention in the expression of collagen-I, $\alpha \mathrm{SMA}$, and TGF $\beta$ in the pirfenidone-treated eyes compared to the PBS-treated eyes. Pirfenidone inhibited increased gene expression of cytokines observed in control eyes. Pirfenidone could be detected up to $48 \mathrm{~h}$ in the vitreous of rabbit eye following single intravitreal injection. Pirfenidone did not show any adverse effect following intravitreal injection; eyes were devoid of any abnormal clinical sign, intraocular pressure, and electroretinography did not show any significant change and histology of retina remained unchanged.

Conclusion This animal study shows that pirfenidone might be a potential therapy for PVR. Further clinical study will be useful to evaluate the clinical application of pirfenidone.

Eye (2017) 31, 1317-1328; doi:10.1038/eye.2017.21; published online 17 March 2017

\section{Introduction}

Proliferative vitreoretinopathy (PVR) is a major sequel of penetrating or open globe injury, with a reported incidence of occurring in $40-60 \%$ of patients with open globe injury. ${ }^{1}$

The high incidence of PVR following ocular trauma is conceived to be due to the inflammatory reaction that follows the injury. Those eyes that develop PVR after a trauma have worse visual outcomes and PVR is considered as the primary reason for the loss of vision. $^{2}$

Recent advances in the fundamental understanding of the pathogenesis of PVR aided in the identification of several new therapeutic targets to block the cellular events contributing to the disease. Cytokines and growth factors are considered to be major players in PVR pathogenesis. ${ }^{3}$

Prospective therapy including antiinflammatory agents, for example, ozurdex, ${ }^{4}$ antineoplastic agents, for example, doxorubicin, ${ }^{5}$ blood clotting factor agents, for example, thrombin ${ }^{6}$ antimetabolite and antiproliferative agents, for example, daunorubicin, ${ }^{7}$

5 -fluorouracil ${ }^{8}$ target one or more of the associated pathologies. Clinical trials assessing the efficacy of antiproliferative and antiinflammatory substances have yielded mixed results. Despite the progress in vitreoretinal surgical techniques, the clinical outcome remains unsatisfactory, and PVR continues to remain a

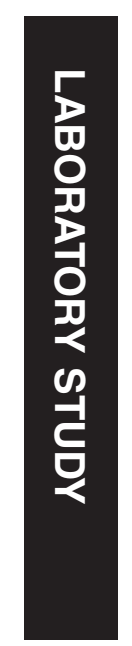

${ }^{1}$ Department of Veterinary Surgery \& Radiology, West Bengal University of Animal \& Fishery Sciences, Kolkata, India

${ }^{2}$ CSIR-Indian Institute of Chemical Biology, Kolkata, India

${ }^{3}$ Disha Eye Hospitals, Kolkata, India

Correspondence:

A Konar, CSIR-Indian Institute of Chemical Biology, Kolkata 700032, India

Tel: +91 33 24995874;

Fax: +913324723963.

E-mail: adityakonar@

yahoo.com

or S Hazra, Department of

Veterinary Surgery \&

Radiology, West Bengal

University of Animal \&

Fishery Sciences, Kolkata

700037, India

Tel: +91 33 25569234;

Fax: +913325571986

E-mail: shazrakon@

yahoo.co.in

${ }^{4}$ These two authors contributed equally to this work.

Received: 27 October 2016 Accepted in revised form: 9 January 2017 Published online: 17 March 2017 
leading cause of blindness. ${ }^{9,10}$ Therefore, more needs to be done to achieve better visual outcome prophylactically and therapeutically in PVR. ${ }^{11,12}$

Pirfenidone is an anti-fibrotic agent approved by US FDA for the treatment of idiopathic pulmonary fibrosis and exhibits promising result for the prevention of liver fibrosis, renal fibrosis, and multiple sclerosis. It has shown inhibition effect on the proliferation, migration, and collagen contraction of human fibroblasts. ${ }^{13}$ In our previous work we have shown prevention of corneal scarring by pirfenidone. ${ }^{14}$ Interestingly pirfenidone has proved to inhibit fibronectin secretion by retinal pigmented epithelial cell (RPE) in vitro. ${ }^{15}$ It inhibits TGF $\beta$ 1 activity in RPE cells by prevention of Smad translocation into the nucleus, ${ }^{16}$ prevents EMT of RPE cells, and has demonstrated no cytotoxicity. ${ }^{17}$

Pirfenidone may, therefore, play a potential role in the prevention of fibrotic changes involved in PVR, which has not yet been explored. The present study was therefore conceived to evaluate the effect of pirfenidone in an animal model of trauma-induced PVR.

\section{Materials and methods}

\section{Animal experiment}

The animal experiments were carried out with prior permission of Institutional Animal Ethics Committee (Approval No. EC- 67, dated 21 May 2013) registered with CPCSEA (Registration No. 763/03/a/CPCSEA, dated 05 June 2003). The work followed the guidelines of the Association for Research in Vision and Ophthalmology (ARVO). Experiments were conducted on clinically healthy New Zealand White rabbit of either sex weighing about $2 \mathrm{~kg}$. Routine clinical evaluation and ophthalmic examinations were done before the experiment.

\section{A rabbit model of penetrating injury induced PVR and evaluation of pirfenidone for its prevention}

Twelve rabbits were anaesthetised with the intramuscular injection of ketamine hydrochloride (Ketamine 50, Themis, Mumbai, India) and xylazine hydrochloride (Xylazine, Indian Immunologicals Ltd, Hyderabad, India) at the dose rate of 35 and $5 \mathrm{mg} / \mathrm{kg}$, respectively. One eye of each animal was instilled with one drop each of $1 \%$ tropicamide (Tropicacyl, 1\% w/v, Sunways Pvt. Ltd, Mumbai, India) and 1\% Proparacaine (Paracain 0.5\%, Sunways) eye drop. The conjunctiva was aseptically prepared by flushing with 5\% povidone iodine. A $23 \mathrm{~g}$ needle was placed $4 \mathrm{~mm}$ from limbus near supraorbital muscles at about $12^{\circ}$ clock position. It was inserted gently into the vitreous cavity at about $45^{\circ}$ angle to a depth of 0.5 inches. While withdrawing, the needle was bent further posteriorly towards retina, and it was touched causing puncture injury with the sharp tip and smoothly removed. The eyes were immediately examined under indirect ophthalmoscope and having ensured vitreal haemorrhage they were treated intravitreally either with $0.1 \mathrm{ml}$ of phosphate-buffered saline (PBS) or with $0.1 \mathrm{ml}$ of $0.5 \%$ pirfenidone in PBS. A drop of ciprofloxacin (Ciplox eye drop, Cipla Ltd, Mumbai, India) was instilled twice daily in all the eyes for 7 days. Antiinflammatories were avoided to prevent masking of an effect of the drug.

The eyes were examined at 1-day interval under indirect ophthalmoscope, adjusting with a 20D lens for 7 days and after that once a week for 1 month.

Twenty-four hours after inciting injury and at the end of 1 month, fundus photographs of all eyes were obtained (Ultra-widefield (UWF) imaging system) to ensure injury and to assess the progression of PVR formation. The eyes were then enucleated after killing with an overdose of anaesthesia and preserved in $10 \%$ formalin. Gross evaluations of the eyes were conducted by dissecting the eyes along the equator to view the posterior segment. The degree of PVR formation was graded and scored according to Fastenberg scale of classification. ${ }^{18}$ In brief, Grade 0: normal retina, Grade 1: vitreous haze, inner retinal wrinkling, Grade 2: retinal wrinkling, retinal detachment one quadrant, Grade 3: retinal wrinkling, retinal detachment two quadrants, Grade 4: retinal wrinkling, retinal detachment three quadrants, Grade 5: retinal wrinkling, retinal detachment four quadrants. Posterior segments of eyes were preserved in $10 \%$ formalin for histopathology and immunohistochemistry.

\section{Expression of inflammatory cytokines}

Penetrating injury to the posterior segment of rabbit eyes was inflicted following the same procedure mentioned earlier. Either $0.1 \mathrm{ml}$ of $0.5 \%$ pirfenidone or $0.1 \mathrm{ml}$ PBS was injected intravitreally into the eyes immediately after the injury. At different time points (24, 48, and $72 \mathrm{~h}$ ) four pirfenidone and four PBS-injected eyes were enucleated following killing and the vitreous along with retinal tissue was extracted by dissection through the equator of the globe. The vitreous along with retinal tissue was collected in TRIZOL Reagent (Cat No.15596-018, Invitrogen Life Technologies, Waltham, MA, USA) and preserved in $-20^{\circ} \mathrm{C}$ for isolation of RNA.

\section{Real-time PCR (qPCR)}

Total RNA from vitreous of normal-, control-, and pirfenidone-treated eyes using TRIZOL Reagent (Cat No. 15596-018, Invitrogen Life Technologies) following standard protocol. The concentration of RNA was 
determined, and cDNA was transcribed according to the kit protocol (Revert Aid TM first-strand cDNA Synthesis Kit, \#K1622, Fermantas, Waltham, MA, USA) Real-time PCR was performed with IL-6 (F: 5'-GGCTGATAGAAG AAGACGGATG-3', R: 5'-CCATGCCTGTCCAGAGATA AAG-3), TNF $\alpha$ (F: 5'-CCTTCCTCTCCTCAGATGTTTC-3', R: 5'-ACGGGTCAGTCACCAAATC-3), transforming growth factor beta (TGF $\beta$ ) (F: 5'-ATAGTCTTCCTGCCGGTCCT-3', R: 5'-TGGGGAGCTTTATGTGCCAG-3', and GAPDH (F: 5'-TCAACAGCAACTCCCACTCTTCCA-3' R: 5'-ACCCT GTTGCTGTAGCCGTATTCA-3') primers using SYBRR Premix Ex TaqTM (Perfect Real Time), Takara, Otsu, Shiga, Japan, \#PR041A) in a real-time PCR cycler (ABI Prism 7500 Sequence Detection System; Applied Biosystems, Foster City, CA, USA). Relative mRNA levels in each sample were calculated after normalisation to GAPDH mRNA expression using DD CT method.

\section{Detection of pirfenidone from vitreous of rabbit eyes following single intravitreal injection}

About $100 \mu \mathrm{l}$ of $0.5 \%$ pirfenidone or PBS was injected intravitreally into one eye of nine rabbits. At different time points following injections $(24,48,72 \mathrm{~h})$ three eyes at each time point was enucleated following killing, the vitreous was extracted and preserved in $-80{ }^{\circ} \mathrm{C}$ for highperformance liquid chromatography (HPLC).

\section{The method for HPLC}

HPLC was performed using a Shimadzu HPLC system (Kyoto, Japan). All reagents used in the procedure were of HPLC grade. The mobile phase consisted of acetonitrile and water $(23: 77, \mathrm{v} / \mathrm{v})$ containing $0.2 \%$ acetic acid. The mobile phase was filtered through a $0.45 \mu \mathrm{m}$ Millipore filter (Merk Millipore Ltd, Darmstadt, Germany) before use and a flow rate of $1 \mathrm{ml} / \mathrm{min}$ was applied in the system. The detection wavelength for pirfenidone was set at $310 \mathrm{~nm}$.

Pirfenidone was dissolved in methanol and diluted to obtain standard solutions in the concentration range of 100-250 $\mu \mathrm{g}$ per ml methanol. The samples were run, and a standard curve was obtained. Control and treated vitreous were homogenised in $1 \mathrm{ml}$ methanol and centrifuged at $12000 \mathrm{rpm}$ for $10 \mathrm{~min}$. The supernatant was collected in fresh centrifuge tubes and was evaporated to dryness under a gentle stream of nitrogen. The residue was dissolved in $200 \mu \mathrm{l}$ methanol and run in the system for detection of pirfenidone. The standard curve was linear, and the equation relating to the area of peaks $(Y)$ against concentration of pirfenidone $(X)$ was $Y=1.1007 X+4.0657$ and $r^{2}=0.98001$.

\section{Evaluation of safety following intravitreal injection of} $0.5 \%$ pirfenidone

For assessment of the safety of intravitreal injection of pirfenidone, one eye of six rabbits was intravitreally injected with $0.1 \mathrm{ml} 0.5 \%$ pirfenidone and the other eye was injected with $0.1 \mathrm{ml}$ of PBS under general and topical anaesthesia as described previously. The eyes were examined on alternate days under slit lamp biomicroscope for signs of inflammation, uveitis, aqueous flare, opacity, and the fundus of the eye was examined by indirect ophthalmoscopy adjusting with a 20D lens on an alternate day. Intraocular pressure (IOP) was measured, and electroretinography (ERG) was performed. After 14 days the rabbits were killed with an overdose of ketamine HCL and xylazine HCL, eyes were enucleated, and the retinas were preserved in $10 \%$ formalin for histopathology.

\section{Assessment of IOP}

IOP was measured before injection of $0.5 \%$ pirfenidone and on 2nd, 7th, and 14th day following injection for evaluation of safety. One drop of proparacaine hydrochloride $(0.5 \%)$ was applied topically on both eyes of each rabbit and IOP was measured using Tonopen (Reichert, Depew, NY, USA), three consecutive readings were taken and averaged. Mean IOP of six eyes is presented.

\section{ERG}

Electroretinography was performed before intravitreal injection of $0.5 \%$ pirfenidone and after 14 days of injection for evaluation of safety following standard procedure. The rabbits were dark-adapted for $2 \mathrm{~h}$, and intermittent instillation with $1 \%$ tropicamide was performed topically on both eyes for pupillary dilatation. They were anaesthetised with an intramuscular injection of xylazine hydrochloride and ketamine hydrochloride at the dose rate of 5 and $35 \mathrm{mg} / \mathrm{kg}$, respectively. One drop of $1 \%$ proparacaine and one drop of methylcellulose (Appavisc 2\% w/v; Appasamy ocular devices, Pvt. Ltd (Pharma Division), Vadamangalam, India) was applied topically on both eyes. ERG recordings were conducted as recommended by the ISCEV guidelines ${ }^{19}$ (Reti Animal, Roland Consultancy, Brandenburg-an der Havel, Germany). The body temperature of the animals was maintained at $37^{\circ} \mathrm{C}$ throughout the experiment. The electrodes were placed under dim red light. The active corneal jet electrodes were put on the cornea, and the reference electrode (subdermal needle electrode) was placed subcutaneously between the base of the ear and 
lateral canthus. The ground electrode was placed subcutaneously just on the occiput (on top of the head).

\section{Histology}

Histopathology was carried out following standard procedure. The tissues were cleared in xylene, dehydrated in graded alcohol, and embedded in paraffin. Sections of $5 \mu$ were cut and stained with haematoxylin-eosin.

\section{Immunohistochemistry}

Vitreoretinal tissue sections were deparaffinized in xylene, rehydrated in graded alcohol and subjected to antigen retrieval by immersing in $10 \mathrm{mM}$ sodium citrate buffer $\mathrm{pH} 6.0$ for $10 \mathrm{~min}$ at $100{ }^{\circ} \mathrm{C}$ in a water bath. Endogenous peroxidase activity was blocked using 3\% hydrogen peroxide for $10 \mathrm{~min}$ and overlaid with blocking buffer ( $5 \%$ BSA) at room temperature for $2 \mathrm{~h}$. After washing in PBST, sections were incubated overnight at $40{ }^{\circ} \mathrm{C}$ with collagen-I $(1: 50$, Abcam, Cambridge, MA, USA; ab90395) or alpha smooth muscle actin ( $\alpha$ SMA) (1 : 50, Abcam; ab7817) or TGF $\beta$ (1:50, Sigma; AV37156) primary antibody. Sections were washed thrice, $5 \mathrm{~min}$ each, in PBST and incubated with secondary antibody (1:200, Goat anti-mouse secondary antibody Alexa flour 488 conjugate, Thermo Fisher Scientific, Waltham, MA, USA, Cat No. A-11001 or Goat anti-mouse IgG secondary antibody Alexa flour 568 conjugate, Thermo Fisher Scientific, Cat No. A-11004). Then the sections were washed and finally stained with DAPI, mounted with a cover slip, and examined under a fluorescence microscope (Leica DM 2500, Leica Microsystems, Wetzlar, Germany).

\section{Statistical analysis}

Statistical analysis was performed with Graph Pad Prism 6 (La Jolla, CA, USA). Data were expressed as mean \pm SD or as indicated. Analysis of PVR grades was conducted by non-parametric Mann-Whitney $U$-test, and for evaluation of safety IOP, ERG data were analysed using $t$-test. Confidence level was set at $95 \%(P<0.05)$.

\section{Results}

\section{Model resembled the clinical PVR}

In the present study retinal injury was induced to rabbit eye to mimic the clinical pathology associated with PVR and in particular PVR arising from an ocular injury. Fundus photographs of the normal retina (Figure 1a) and injured retina (Figure $1 \mathrm{~b}$ ) taken $24 \mathrm{~h}$ after induction are presented. Fundus photograph of injured eye clearly showed injury in the retina resulting in a breach of continuity and profuse haemorrhage (Figure 1b), whereas the normal retina was without these changes. Fundus photograph, taken 1 month after induction, showed wrinkling of the retina, retinal detachment, and epiretinal membrane formation (Figure 1c). The posterior view showed folds in the retina and fibrosis (Figure 1d). Prominent retinal scar, retinal detachment, and epiretinal membrane formation was observed in the histopathological sections. These changes are characteristic features of clinical cases of PVR. Thus, the model developed in the present study completely mimicked the clinical pathogenesis and pathology of PVR.

\section{Pirfenidone prevented PVR in the animal model of penetrating injury}

One month after penetrating injury and pirfenidone treatment, eyes were evaluated by fundus photography as well as by posterior view for gross pathology. The PVR in different eyes was graded and scored. A significant $(P=0.0003)$ decrease in PVR score was observed in the pirfenidone-treated group $(0.78 \pm 0.44)$ compared to untreated controls $(2.33 \pm 0.86)$. Control eyes showed several folds in the retina, distinct retinal detachment, and epiretinal membrane formation (Figures $1 \mathrm{c}$ and $\mathrm{d}$ ). The pirfenidone-treated eyes were devoid of such severe changes and showed only slight retinal fold and mild vitreous haze (Figures 1e and f).

\section{Histopathology showed prevention of PVR in pirfenidone-treated eyes}

Histopathology of control eyes showed a prominent scar in the retina, a retinal detachment was also evident, and an epiretinal membrane was observed (Figures 2a, $\mathrm{a}^{*}$, and $b$ ). While in the pirfenidone-treated group changes like severe fibrosis, epiretinal membrane, and retinal detachment were absent; however mild to moderate inflammatory cellular proliferation was evident in all eyes (Figure 2c).

\section{Pirfenidone downregulates the expression of IL-6, TNFa, and TGF $\beta$}

RT-PCR was performed to evaluate the expression of IL-6, $\mathrm{TNF} \alpha$, and TGF $\beta 1$ the predominant cytokines and growth factor involved in PVR pathogenesis. A significant $(P<0.05)$ increase in the expression of IL-6, and TNF $\alpha$ was observed at different time points in the vitreoretinal tissue from rabbit eyes with a penetrating injury. A significant $(P<0.05)$ decrease in mRNA expression of IL-6, TNF $\alpha$ (Figure 3) was observed in pirfenidone-treated eyes compared to untreated control eyes at similar time points. 
a

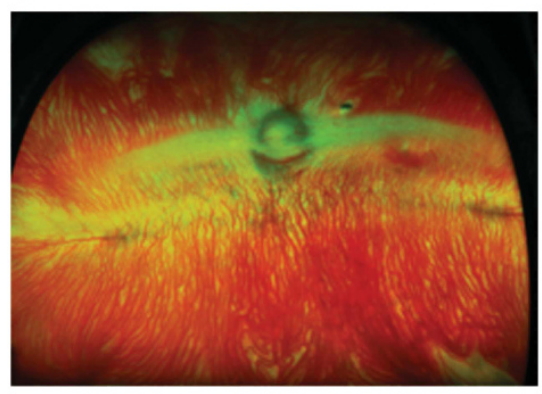

c

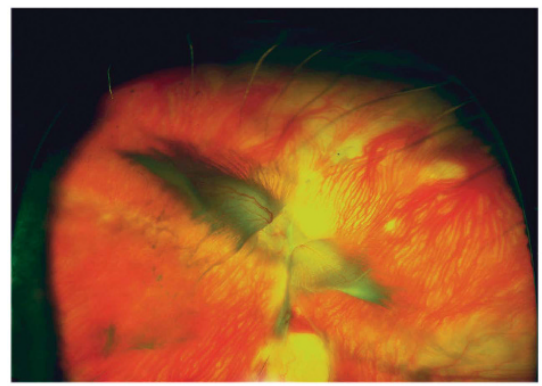

e

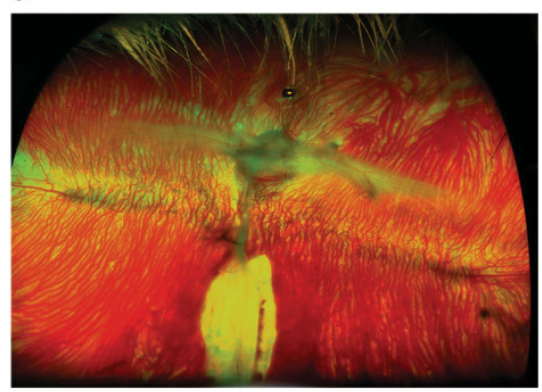

b

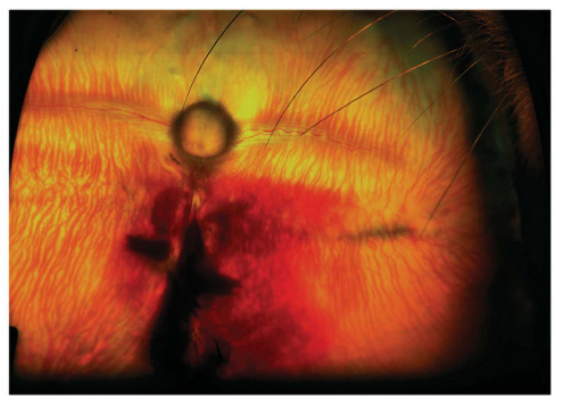

d

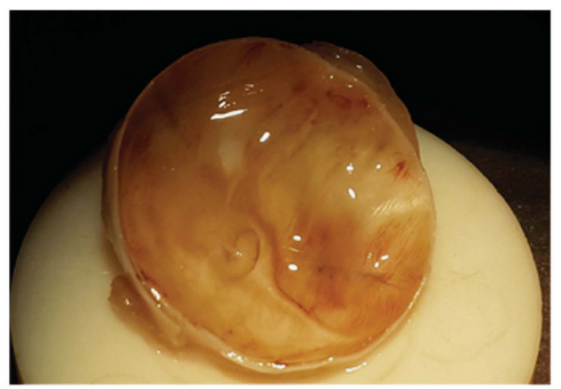

f

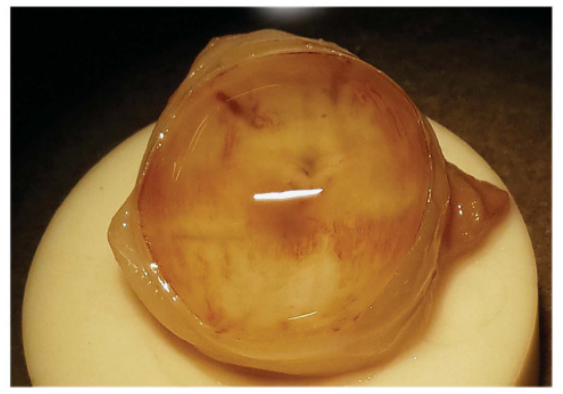

Figure 1 A penetrating injury was inflicted on the retina of a rabbit. After $24 \mathrm{~h}$ of injury, fundus was examined and compared with the fundus of normal rabbit. Fundus photograph from injury-induced animal shows a breach of continuity and profuse haemorrhage (b) whereas the normal retina is devoid of such changes and shows merangiotic vasculature and optic nerve head (a). Pirfenidone reduces retinal folding and detachment: at 1 month post induction, fundus photograph (c) and gross posterior segment view (d) of PBS-treated control eye shows retinal traction, folding, and detachment; whereas the fundus photograph (e) and gross posterior view (f) of pirfenidone-treated eye shows absence of these PVR signs, that is, retinal traction, folding, and detachment.

Expression of TGF $\beta$ was also noted to be increased significantly $(P<0.05)$ in the control animals, which was reduced in the pirfenidone-treated eyes (Figure 3).

\section{Pirfenidone prevents fibrotic changes}

EMT of RPE cells resulting in fibrosis and epiretinal membrane formation are the hallmark PVR. Therefore, expression of $\alpha \mathrm{SMA}$ and collagen-I in the histology sections was evaluated. Immunohistochemistry showed prominent expression of $\alpha \mathrm{SMA}$ in the control eyes (Figure $4 \mathrm{a}$ ), whereas a negligible expression of $\alpha \mathrm{SMA}$ was observed in the sections from pirfenidone-treated eyes (Figure 4c). Accordingly, increased expression of collagen-I was evidenced in the control sections (Figure 4e), whereas the expression of collagen-1 was minimum in sections from pirfenidone-treated eyes (Figure 4g). An increased expression of TGF $\beta$ was observed in sections from the injury-inflicted control eyes (Figure 4i) and a reduction in the expression of TGF $\beta$ was evidenced in the pirfenidone-treated eyes (Figure 4k).

\section{Pirfenidone was available in the vitreous}

Pirfenidone was evaluated in the vitreous humour of rabbits at different time points by HPLC following single intravitreal injection. The drug could be detected up to $48 \mathrm{~h}$ after injection. The level (mean $\pm \mathrm{SE}$, in $\mu \mathrm{g}$ ) of pirfenidone detected at 24 and $48 \mathrm{~h}$ was $214.9 \pm 78.84$ and $29.45 \pm 7.15$, respectively. 
a

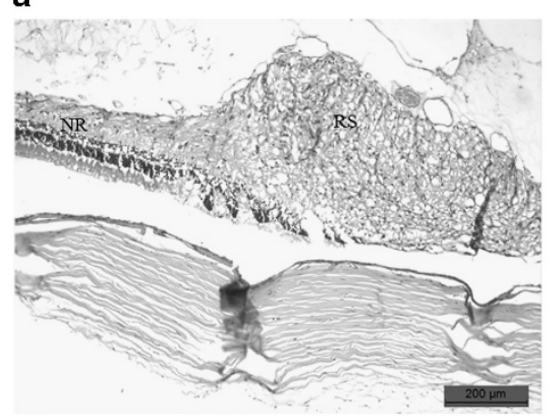

b

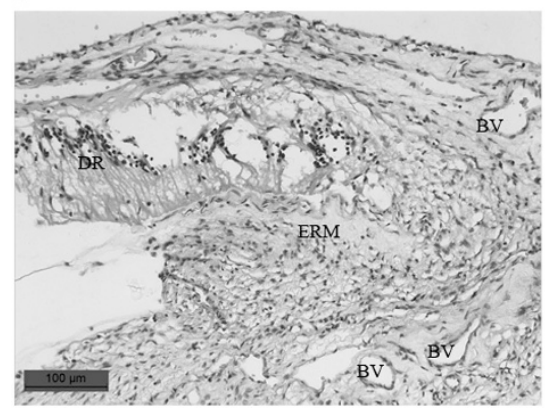

$a^{*}$

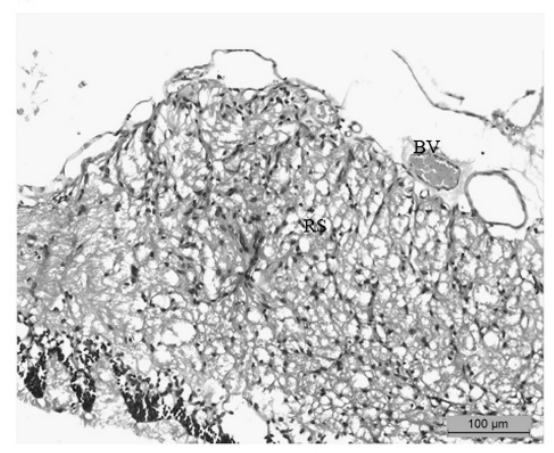

C

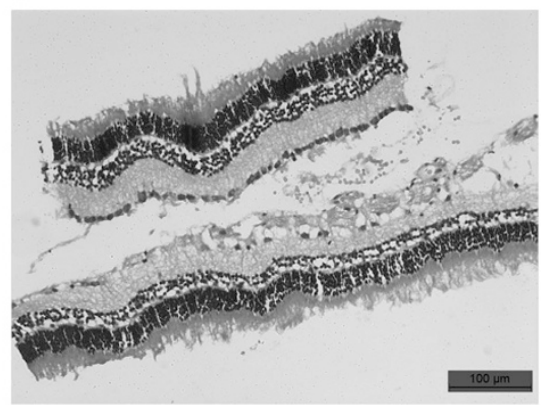

Figure 2 (a) Histology section of control retina shows prominent retinal scar and a portion of normal retina (NR), ( $\left.\mathrm{a}^{*}\right)$ prominent retinal scar at 1 month post induction highlighted. (b) Another section of retina from control eye shows the presence of epiretinal membrane (ERM), detachment of retina, and blood vessels (BV). (c) In the section from the pirfenidone-treated retina, scar, retinal detachment, or epiretinal membrane formation was absent. However, infiltration of inflammatory cells was observed.

\section{Pirfenidone did not show any adverse effect}

Pirfenidone was injected into the vitreous humour of normal rabbit eyes and following observations were made to evaluate any adverse effect.

\section{Indirect ophthalmoscopy}

No adverse effect was observed following intravitreal injection of pirfenidone. There was no sign of vitreous haemorrhage, haze, or retinal detachment when examined on 1st, 7 th, and 14th day following treatment.

\section{Slit lamp biomicroscopy}

Examination under slit lamp biomicroscope showed clear transparent anterior segment in the pirfenidone-treated eyes without any sign of inflammatory response or evidence of uveitis, that is, aqueous flare. Corneal or lens opacity was also absent. None of the eyes suffered from subconjunctival haemorrhage (Supplementary Figure 1A). The eye resembles the PBS-treated eyes (Supplementary Figure 1B).

\section{Intraocular pressure}

There was no significant difference in IOP (mean \pm SE) following intravitreal injection of $0.1 \mathrm{ml}$ of $0.5 \%$ pirfenidone on 2 nd $(8.5 \pm 0.6$ vs $8.27 \pm 0.74)$, 7 th $(8.05 \pm 0.82$ vs $7.77 \pm 1.06)$, and 14 th $(8.04 \pm 0.86$ vs $7.89 \pm 0.71)$ day compared to PBS-treated control eyes as well as pretreatment baseline values $(8.83 \pm 0.86)$.

\section{ERG}

The electroretinography of pirfenidone-treated eyes resembled the normal eyes. The amplitude of $a$ wave and $b$ waves with the different stimulus of scotopic and photopic tests did not vary significantly in comparison to pretreatment baseline values (Table 1).

\section{Histopathology}

Histopathological sections of the retina from pirfenidonetreated eyes (Supplementary Figure 1C) did not show any adverse change and resembled the architecture of the normal retina. (Supplementary Figure 1D). 


\section{Discussion}

PVR is a leading cause of blindness following retinal reattachment surgery, ocular trauma, and primary retinal detachment. Despite the tremendous advancement in vitreoretinal surgery and therapeutic approaches for management of PVR, it continues to remain a clinical challenge. The incidence of ocular trauma is alarming, and PVR follows 40-60\% of all penetrating ocular injuries.
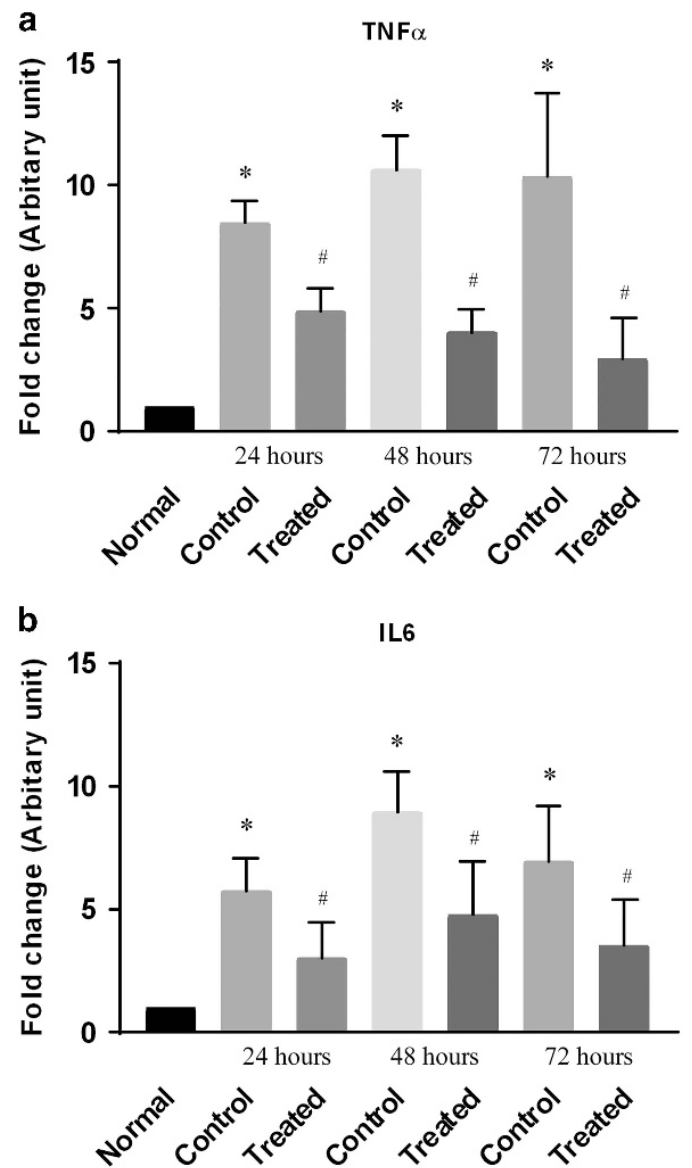

c

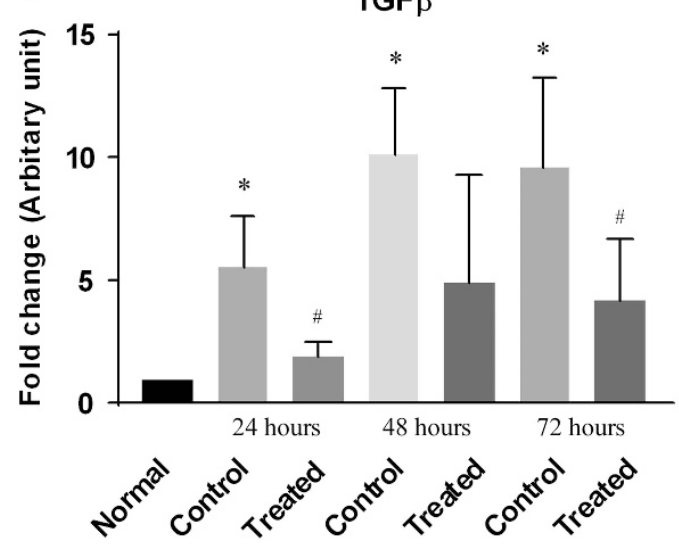

In the present study, we have established a model of PVR in rabbit by inducing intravitreal injury. Several models of PVR have been developed by delivering either a piece of dermal tissue, ${ }^{20}$ fibroblast, $^{21-23}$ RPE cells, ${ }^{24}$ or platelet and IL- $1 \beta^{25}$ into the vitreous humour of rabbit or rat. Although these models develop vitreous strands and retinal detachment, the injection of a particular type of cell does not depict the effect of other cell types involved in PVR pathology. Therefore to mimic the etiopathogenesis of PVR more closely, we have developed this model by inciting retinal injury. We observed intravitreal haemorrhage, a tear in the retina, and an inflammatory response as commonly met with in clinical settings such as accidental needle prick or during retrobulbar injection, injury due to sharp stick or sharp pencil. The eyes developed PVR characterised by retinal detachment and epiretinal membrane formation. The trauma model of PVR used currently ${ }^{26}$ is created by causing a prolapse of the vitreous by placing scleral incision, followed by injection of autologous blood. It does not mimic the clinical presentation as it is assumed that cells local to the retina and vitreous are more closely associated with the pathogenesis than the cells introduced from outside.

In the present study, we observed prevention of PVR following intravitreal use of pirfenidone. The retinal detachment, as well as epiretinal membrane formation in pirfenidone-treated eyes, was significantly lower compared to control.

Three predominant events associated with fibrogenesis are oxidative stress, inflammation, and eventual fibrosis. The presence of cytokines IL- $1,{ }^{27} \mathrm{IL}-6,{ }^{28} \mathrm{TNF} \alpha,{ }^{28,29} \mathrm{IFN} \gamma,{ }^{28}$ IL- $8,{ }^{28}$ IL-10, ${ }^{28,30}$ and growth factors FGF, ${ }^{28}$ PDGF, ${ }^{31}$ $\mathrm{HGF}^{31}$ are evidenced in PVR and therefore are associated with the PVR pathogenesis. Experimental results, as well as clinical cases of PVR, have identified IL-6 $6^{32-34}$ and TNF $\alpha^{34-37}$ as major players in PVR pathogenesis. TGF $\beta$ plays a critical role in PVR development. ${ }^{33,34,38-41}$ Extensive studies have set forth the molecular

Figure 3 Pirfenidone prevents cytokine expression. The ocular injury was inflicted in rabbit eyes and injected intravitreally either with PBS (control) or pirfenidone, expression of cytokines in the vitreous of normal, control (PBS treated ) and pirfenidonetreated eyes were compared. (a) Bar diagram shows significant $(P<0.05)$ increase in IL-6 expression in the vitreous of control eyes compared to normal eyes at different time points and its suppression in the vitreous of pirfenidone-treated eyes. (b) Bar diagram shows significant $(P<0.05)$ increase in TNF $\alpha$ expression in the vitreous of control eyes at different time points and its prevention in the vitreous of pirfenidone-treated eyes. (c) Bar diagram shows significant $(P<0.05)$ increase in the expression of TGF $\beta$ in the vitreous of control eyes and its prevention in pirfenidone-treated eyes. Data expressed as mean $\pm \mathrm{SD}, N=3$, ${ }^{*} P<0.05$ vs Normal, ${ }^{\#} P<0.05$ vs Control. 
mechanisms of pirfenidone in arresting fibrosis, which it does by ameliorating oxidative stress, inhibiting major inflammatory cytokines contributing to fibrosis, that is,
TNF $\alpha$, IL-6, and specifically inhibiting growth factor, TGF $\beta .^{42}$ In the present study, we observed an increase in IL-6 and TNF $\alpha$ in control eyes at different time a

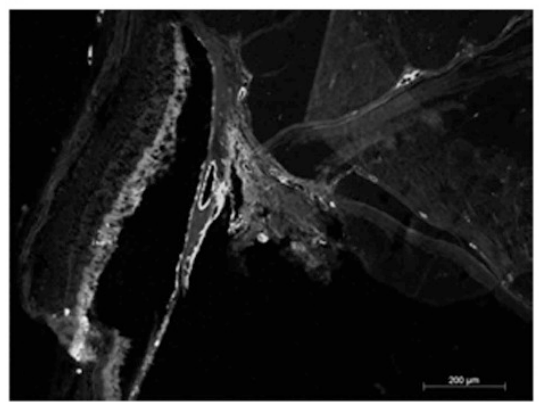

d

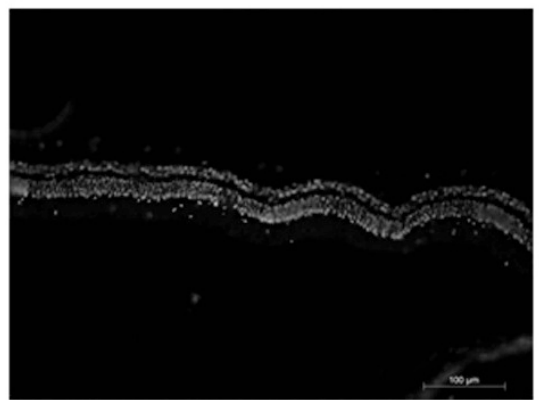

g

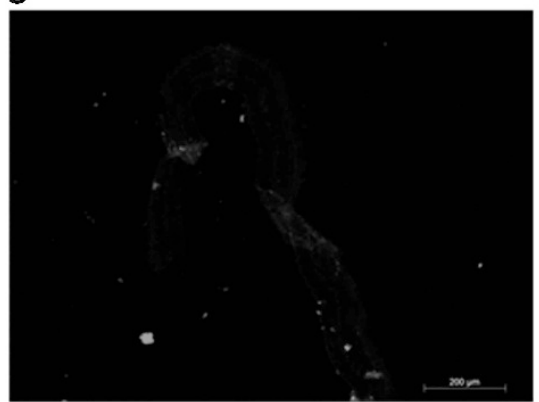

j

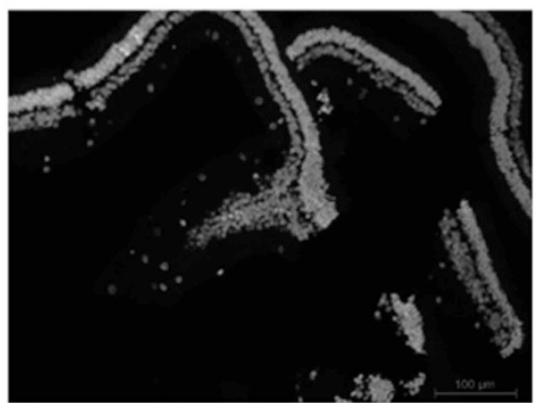

b

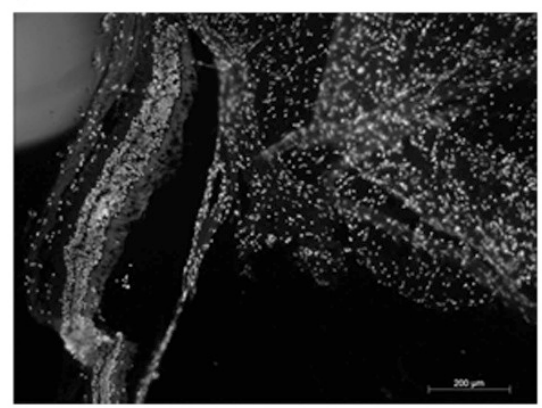

e

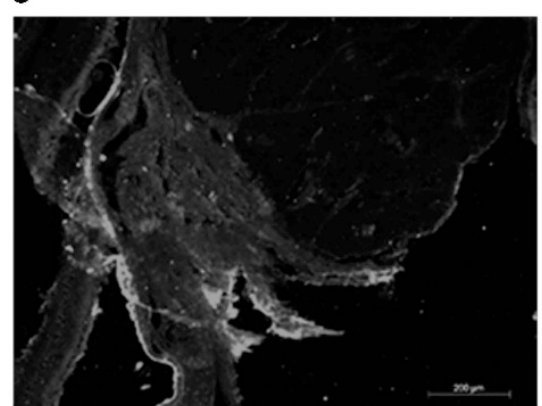

h

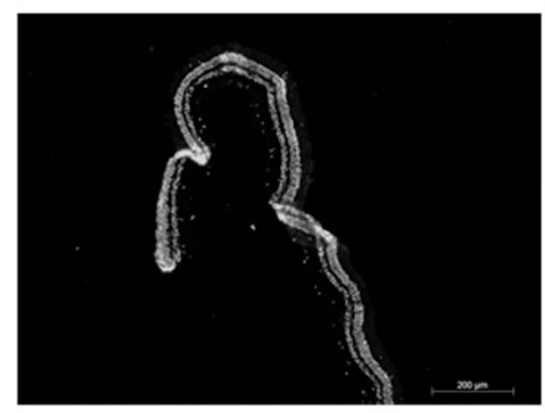

k

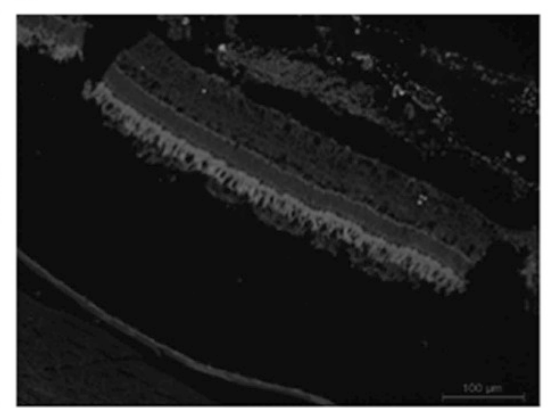

C

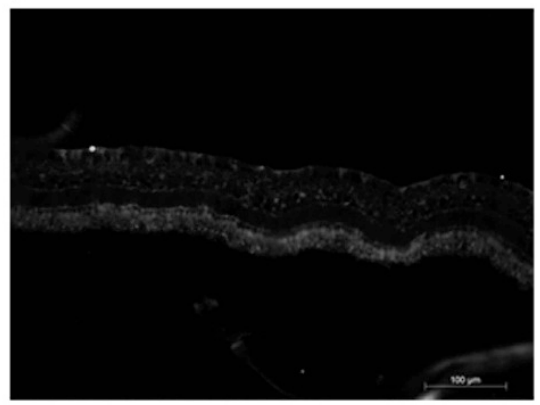

f

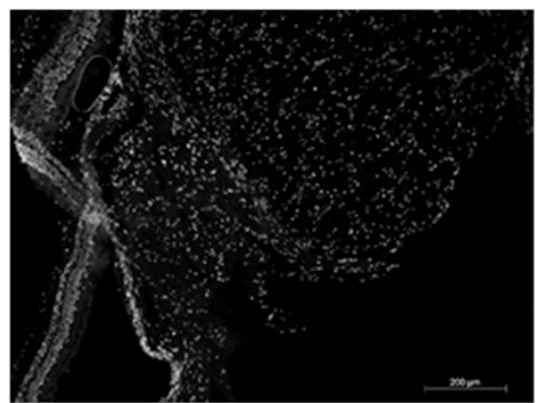

i

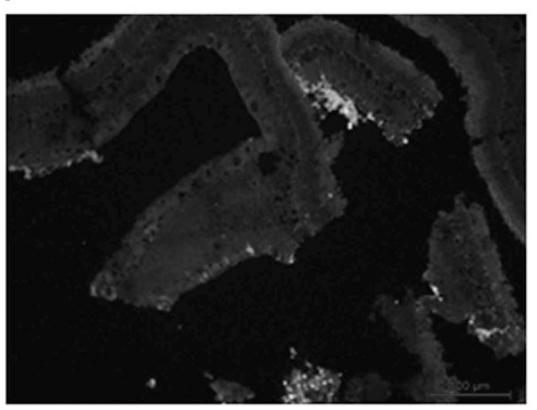

I

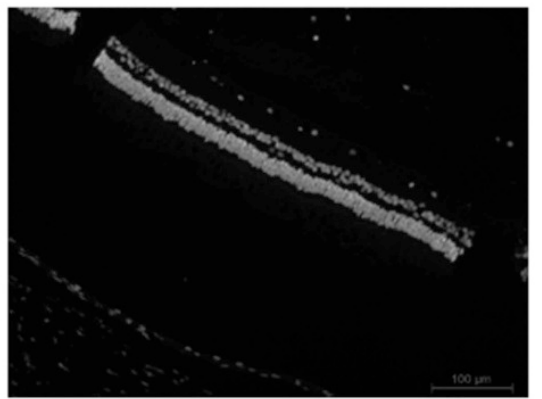

Figure 4 Pirfenidone suppressed the expression of collagen-I, $\alpha \mathrm{SMA}$, and TGF $\beta$. A penetrating injury was inflicted to the posterior section of a rabbit eye and intravitreally injected either with pirfenidone or PBS. After 1 month expressions of collagen-I, $\alpha$ SMA, and TGF $\beta$ were evaluated. Immunohistochemistry of control sections showed huge expression of collagen-I (a), (DAPI-stained section (b)), compared to sections from pirfenidone-treated sections (c), (DAPI-stained section (d)). Increased expression of $\alpha$ SMA was observed in the control sections (e) (DAPI-stained section (f)) and its absence in the sections from pirfenidone-treated sections (g), (DAPI-stained sections (h)). Control sections (i) (DAPI-stained section (j)) showed increased expression of TGF $\beta$ and suppression of its expression in pirfenidone-treated sections (k) (DAPI-stained section (l)). 
Table 1 ERG values of pre-treated and pirfenidone-treated eyes

\begin{tabular}{|c|c|c|c|c|}
\hline & \multicolumn{2}{|c|}{$\mathrm{b}$ wave amplitude } & \multicolumn{2}{|c|}{ a wave amplitude } \\
\hline & Pirfenidone-treated & Pre-treated value & Pirfenidone-treated & Pre-treated value \\
\hline Scotopic 0.1 & $161.8 \pm 49.28$ & $187.2 \pm 42.6$ & & \\
\hline Scotopic 3.0 & $311.0 \pm 61.85$ & $262 \pm 51.79$ & $96.04 \pm 5.88$ & $109.78 \pm 16.36$ \\
\hline Scotopic 10 & $306.6 \pm 85.10$ & $256.2 \pm 100.48$ & $120.98 \pm 11.63$ & $120.94 \pm 18.31$ \\
\hline Photopic 3.0 & $140.26 \pm 60.02$ & $144.4 \pm 33.26$ & $7.21 \pm 1.30$ & $6.88 \pm 1.28$ \\
\hline \multirow[t]{2}{*}{ Photopic $30 \mathrm{~Hz}$ flicker N1/P1 } & $\mathrm{N} 1 / \mathrm{P} 1$ & & & \\
\hline & $92.64 \pm 36.40$ & $106.1 \pm 31.05$ & & \\
\hline
\end{tabular}

Data are presented as mean \pm SE $(N=5)$.

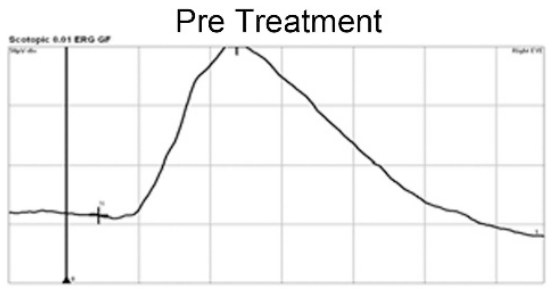

Post Treatment
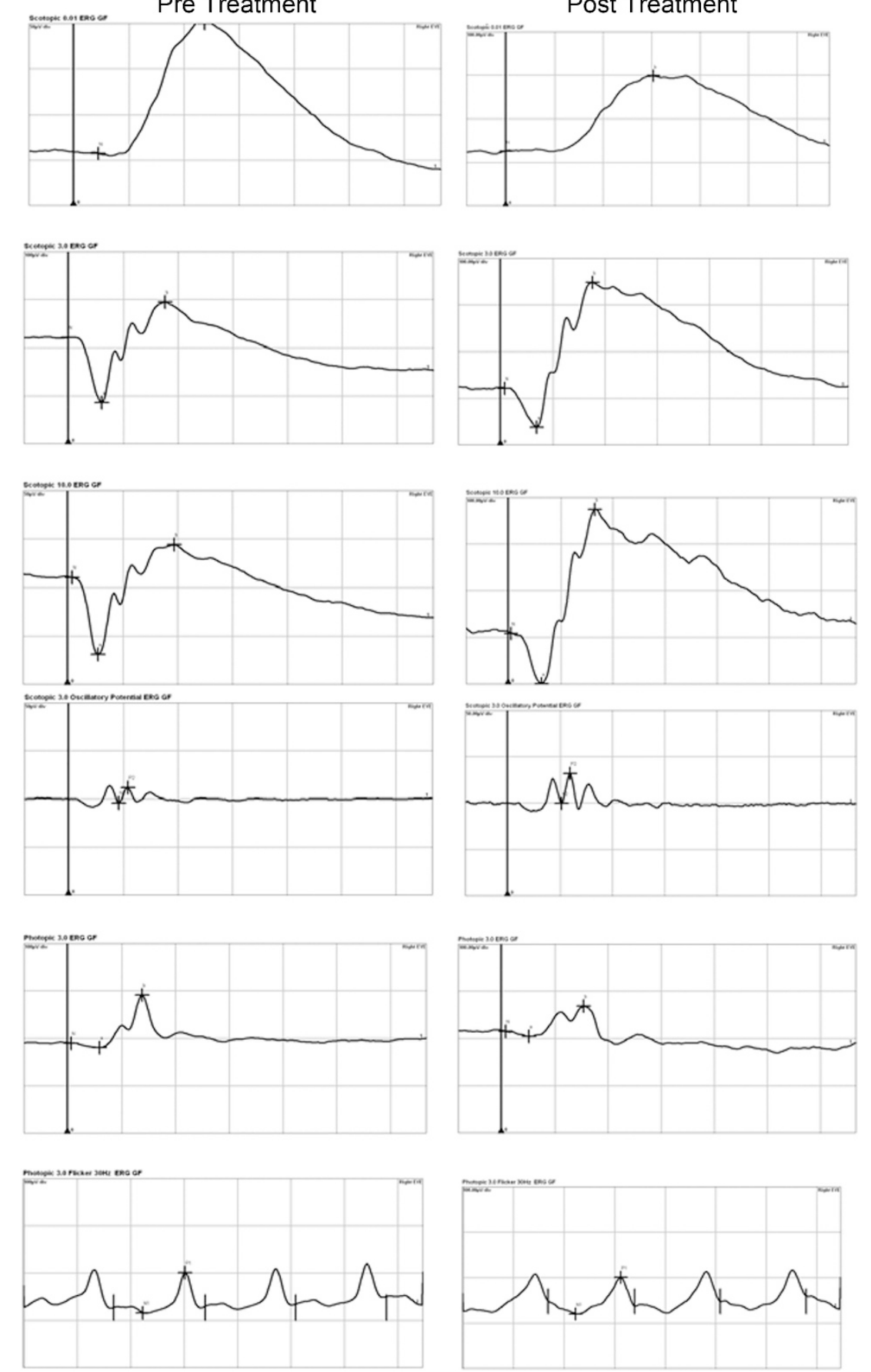
points, which were prevented in pirfenidone-treated eyes; similar modulation of cytokines with pirfenidone was also documented in patients with chronic hepatitis. ${ }^{43}$ Pirfenidone also suppressed the expression of TGF $\beta$ in treated eyes compared to controls. In our earlier study, we have reported suppression of TGF $\beta$ and prevention of EMT in the cornea by pirfenidone. ${ }^{14}$ Wang et al ${ }^{17}$ have shown pirfenidone prevents EMT in RPE cells in vitro. Prevention of EMT and fibrosis has also been documented in lungs and kidney cells with pirfenidone. ${ }^{44-46}$ Taken together our results show the suppression of inflammatory cytokine and TGF $\beta$ by pirfenidone has contributed to the prevention of PVR.

In the clinical setting, it may not be expected for patients to report immediately following penetrating injury in the eye that raises a pertinent question if intravitreal pirfenidone may be beneficial within $24-48 \mathrm{~h}$ following injury. Given the observation that the posttrauma inflammation in the retina persists up to $72 \mathrm{~h}$ and application of agents having anti-inflammatory effect following penetrating injury prevented PVR through suppression of cytokine, ${ }^{47}$ pirfenidone is likely to offer protection if used within $24-48 \mathrm{~h}$. However, this needs further investigation. In patients undergoing surgery for retinal reattachment, triamcinolone acetonide is commonly used along with silicon oil tamponade to prevent inflammation. ${ }^{48}$ We assume intravitreal injection of pirfenidone immediately following vitrectomy prior to silicon oil/gas injection and even at the time of silicon oil removal may also be beneficial in PVR prevention. In addition, it may also enable early silicon oil removal and prevent PVR formation that follows. ${ }^{49}$

Pirfenidone was available in the vitreous humour for $48 \mathrm{~h}$ following single intravitreal injection that almost covered the initial inflammatory phase ${ }^{29}$ after injection, which is very critical for the development of PVR. Pirfenidone suppressed the cytokine expression in the vitreoretinal tissue up to $72 \mathrm{~h}$, which indicates it might have been distributed and remained within the retinal tissue. There is a paucity of literature on the pharmacokinetics of pirfenidone following local use in the eye. The sole study conducted so far demonstrates wide distribution among ocular tissues (including into the vitreous humour) and quick clearance of the drug following topical instillation. ${ }^{50}$ This corroborates with the quick clearance of pirfenidone from vitreous in our study, as the availability of the drug was not evaluated in the retinal tissues, we assume it may have distributed locally to the retina and persisted for some more time offering the anti-inflammatory effect, that is, up to $72 \mathrm{~h}$, beyond its period of availability in vitreous, that is, $48 \mathrm{~h}$. The gene expressions of cytokines were detected from combined vitreous and retinal tissues. However considering the rapid clearance of pirfenidone, we believe a sustained release formulation may prevent the need for repeated intravitreal injections, and with advances in ocular drug delivery a formulation of pirfenidone to deliver a therapeutic dose of the drug to the back of the eye following topical application may be of potential value.

A single intravitreal dose of pirfenidone did not incite any toxicity to the eye. Use of steroids though effective for prevention of PVR is associated with the adverse effects such as increase in IOP, cataract formation, subconjunctival haemorrhage. ${ }^{51}$ 5-flurouracil and lower molecular weight heparin are currently in use for PVR prevention, but the intravitreal injection of 5-flurouracil causes prolonged corneal oedema. ${ }^{52}$ In the present study, we did not observe any adverse effect following intravitreal injection of pirfenidone; the eyes were clinically healthy, IOP and electrophysiology of the retina remained unchanged. Topical application of pirfenidone has previously shown to be non-toxic to the eyes. ${ }^{53}$ It has been approved by FDA for clinical use in respiratory diseases ${ }^{54}$ and is being used in some countries.

The results of our work demonstrate intravitreal therapy with pirfenidone as a potential and safe approach for the prevention of PVR following an ocular injury. Further clinical study will be useful to evaluate the clinical application of pirfenidone.

\section{Summary}

What was known before

- Pirfenidone prevents EMT in retinal pigmented epithelial cells in vitro.

What this study adds

- This study adds extensive in vivo data of proliferative vitreoretinopathy (PVR) prevention with pirfenidone in animal model of traumatic PVR. To our knowledge this is the first study that demonstrates safety of intravitreal use of pirfenidone.

\section{Conflict of interest}

The authors declare no competing financial interests.

\section{Author contributions}

Conceived and designed the experiments: $\mathrm{SH}, \mathrm{AK}$. Performed the experiments: BNMKK, VPS, SN, RG, SH. Analyzed the data: $\mathrm{SH}, \mathrm{AK}$. Contributed reagents/ materials/analysis tools: $\mathrm{SH}, \mathrm{AK}, \mathrm{SKB}$. Wrote the paper: $\mathrm{SH}, \mathrm{AK}$. 


\section{Acknowledgements}

We acknowledge the Department of Science \& Technology, DST for funding, and West Bengal University of Animal \& Fishery Sciences, CSIR-Indian Institute of Chemical for providing facilities.

\section{References}

1 Colyer MH, Weber ED, Weichel ED, Dick JS, Bower KS, Ward TP et al. Delayed intraocular foreign body removal without endophthalmitis during operations Iraqi freedom and enduring freedom. Ophthalmology 2007; 114: 1439-1447.

2 Meitz H, Heimann K. Onset and recurrence of proliferative vitreoretinopathy in various vitreoretinal disease. $\mathrm{Br} J$ Ophthalmol 1995; 79: 874-877.

3 Sadaka A, Giuliari GP. Proliferative vitreoretinopathy: current and emerging treatments. Clin Ophthalmol 2012; 6: 1325-1333.

4 Banerjee PJ, Bunce C, Charteris DG. Ozurdex(R) (a slowrelease dexamethasone implant) in proliferative vitreoretinopathy: study protocol for a randomized controlled trial. Trials 2013; 14: 358.

5 Kuo HK, Chen YH, Wu PC, Wu YC, Huang F, Kuo CW et al. Attenuated glial reaction in experimental proliferative vitreoretinopathy treated with liposomal doxorubicin. Invest Ophthalmol Vis Sci 2012; 53: 3167-3174.

6 Bastiaans J, van Meurs JC, van Holten-Neelen C, Nagtzaam NMA, van Hagen PM, Chambers RC et al. Thrombin induces epithelial-mesenchymal transition and collagen production by retinal pigment epithelial cells via autocrine PDGFreceptor signalling. Invest Ophthalmol Vis Sci 2013; 54: 8306-8314.

7 Khawly JA, Saloupis P, Hatchell DL. Daunorubicin treatment in a refined experimental model of proliferative vitreoretinopathy. Graefes Arch Clin Exp Ophthalmol 1991; 229: 464-467.

8 Sundaram V, Barsam A, Virgili G. Intravitreal low molecular weight heparin and 5-fluorouracil for the prevention of proliferative vitreoretinopathy following retinal reattachment surgery. Cochrane Database Syst Rev 2010; (7): CD006421.

9 Cardillo JA, Timothy SJ, LaBree L, Stanley MS, Azen P, Omphroy L et al. Post-traumatic proliferative vitreoretinopathy. Ophthalmology 1997; 104: 1166-1173.

10 Pennock S, Haddock LJ, Eliott D, Mukai S, Kazlauskas A. Is neutralising vitreal growth factors a viable strategy to prevent proliferative vitreoretinopathy? Prog Retin Eye Res 2014; 9: 34-37.

11 Pastor JC, Rojas J, Pastor-Idoate S, Di Lauro S, Gonzalez-Buendia L, Delgado-Tirado S. Proliferative vitreoretinopathy: a new concept of disease pathogenesis and practical consequences. Prog Retin Eye Res 2015; 51: 125-155.

12 Khan MA, Brady CJ, Kaiser RS. Clinical management of proliferative vitreoretinopathy: an update. Retina 2015; 35: 165-175.

13 Lin X, Yu M, Wu K, Yuan H, Zhong H. Effects of pirfenidone on proliferation, migration, and collagen contraction of human Tenon's fibroblasts in vitro. Invest Ophthalmol Vis Sci 2009; 50: 3763-3770.

14 Chowdhury S, Guha R, Trivedi R, Kompella UB, Konar A, Hazra S. Pirfenidone nanoparticles improve corneal wound healing and prevent scarring following alkali burn. PLoS One 2013; 8: e70528.

15 Zhang S, Shiels IA, Ambler JS, Taylor SM. Pirfenidone reduces fibronectin synthesis by cultured human retinal pigment epithelial cells. Aust NZ J Ophthalmol 1998; 1: S74-S76.

16 Choi K, Lee K, Ryu SW, Im M, Kook KH. Pirfenidone inhibits transforming growth factor- $\beta 1$-induced fibrogenesis by blocking nuclear translocation of Smads in human retinal pigment epithelial cell line ARPE-19. Mol Vis 2012; 18: 1010-1020.

17 Wang J, Yang Y, Xu J, Lin X, Wu K, Yu M. Pirfenidone inhibits migration, differentiation, and proliferation of human retinal pigment epithelial cells in vitro. Mol Vis 2013; 19: $2626-2635$.

18 Fastenberg DM, Diddie KR, Sorgente N, Ryan SJ. A comparison of different cellular inocula in an experimental model of massive periretinal proliferation. Am J Ophthalmol 1982; 93: 559-564.

19 McCulloch DL, Marmor MF, Brigell MG, Hamilton R, Holder GE, Tzekov R et al.. ISCEV standard for full-field clinical electroretinography (2015 update). Doc Ophthalmol 2015; 130: 1-12.

20 Algvere P, Kock E. Experimental fibroplasia in the rabbit vitreous. Retinal detachment induced by autologous fibroblasts. Albrecht Von Graefes Arch Klin Exp Ophthalmol 1976; 199: 215-222.

21 Sugita G, Tano Y, Machemer R. Intravitreal autotransplantation of fibroblasts. Am J Ophthalmol 1980; 89: 121-130.

22 Chandler DB, Quansah FA, Hida T, Machemer R. A refined experimental model for proliferative vitreoretinopathy. Graefes Arch Clin Exp Ophthalmol 1986; 224: 86-91.

23 Hitchins CA, Grierson I. Intravitreal injection of fibroblasts: the pathological effects on the ocular tissues of the rabbit following an intravitreal injection of autologous skin fibroblasts. Br J Ophthalmol 1988; 72: 498-510.

24 Zheng XZ, Du LF, Wang HP. An immunohistochemical analysis of a rat model of proliferative vitreoretinopathy and a comparison of the expression of TGF- $\beta$ and PDGF among the induction methods. Bosn J Basic Med Sci 2010; 10: 204-209.

25 Khoroshilova-Maslova IP, Leparskaya NL, Nabieva MM, Andreeva LD. Experimental modelling of proliferative vitreoretinopathy. An Experimental Morphological Study. Bull Exp Biol Med 2015; 159: 100-102.

26 Nassar K, Lüke J, Lüke M, Kamal M, El-Nabi EA, Soliman M et al. The novel use of decorin in prevention of the development of proliferative vitreoretinopathy (PVR). Graefes Arch Clin Exp Ophthalmol 2011; 249: 1649-1660.

27 Liou GI, Pakalnis VA, Matragoon S. HGF regulation of RPE proliferation in an IL-1 $\beta /$ retinal hole-induced rabbit model of PVR. Mol Vis 2002; 8: 494-501.

28 Banerjee S, Savant V, Scott RAH, Curnow SJ, Wallace GR, Murray PL. Multiplex bead analysis of vitreous humor of patients with vitreoretinal disorders. Invest Ophthalmol Vis Sci 2007; 48: 2203-2207.

29 Hui Y, Shi Y, Zhang X, Yang K, Yu C. TNF-alpha, IL-8 and IL-6 in the early inflammatory stage of experimental PVR model induced by macrophages. Zhonghua Yan Ke Za Zhi 1999; 35: 140-143.

30 Elner SG, Elner VM, Jaffe GJ, Stuart A, Kunkel SL, Strieter RM. Cytokines in proliferative diabetic retinopathy and 
proliferative vitreoretinopathy. Curr Eye Res 1995; 14: 1045-1053.

31 Cui JZ, Chiu A, Maberley D, Ma P, Samad A, Matsubara JA. Stage specificity of novel growth factor expression during development of proliferative vitreoretinopathy. Eye 2007; 21: 200-208.

32 Danny JH, Meurs KJC, Mertens DAE, Peperkamp E, Master $\mathrm{XC}$, Gerritsen ME. Cytokines in vitreous humor: interleukin6 is elevated in proliferative vitreoretinopathy. Invest Ophthalmol Vis Sci 1994; 35: 900-906.

33 Kon CH, Occleston NL, Aylward GW, Khaw PT. Expression of vitreous cytokines in proliferative vitreoretinopathy: a prospective study. Invest Ophthalmol Vis Sci 1999; 40: 705-712.

34 Morescalchi F, Duse S, Gambicorti E, Romano MR, Costagliola C, Semeraro F. Proliferative vitreoretinopathy after eye injuries: an overexpression of growth factors and cytokines leading to a retinal keloid. Mediators Inflamm 2013; 2013: 269787.

35 Armstrong D, Augustin AJ, Spengler R, Al-Jada A, Nickola $\mathrm{T}$, Grus F et al. Detection of vascular endothelial growth factor and tumour necrosis factor alpha in epiretinal membranes of proliferative diabetic retinopathy, proliferative vitreoretinopathy and macular pucker. Ophthalmologica 1998; 212: 410-414.

36 Limb GA, Daniels JT, Pleass R, Charteris DG, Luthert PJ, Khaw PT. Differential expression of matrix metalloproteinases 2 and 9 by glial Muller cells: response to soluble and extracellular matrix-bound tumour necrosis factor-alpha. Am J Pathol 2002; 160: 1847-1855.

37 Rojas J, Fernandez I, Pastor JC. A strong genetic association between the tumour necrosis factor locus and proliferative vitreoretinopathy: the Retina 4 Project. Ophthalmology 2010; 117: 2417-2423.

38 Connor TB, Roberts AB, Sporn MB. Correlation of fibrosis and transforming growth factor- type 2 levels in the eye. J Clin Invest 1989; 83: 1661-1666.

39 Kimoto K, Nakatsuka K, Matsuo N, Yoshioka H. MAPK mediates the expression of type I collagen induced by TGF-2 in human retinal pigment epithelial cells ARPE-19. Invest Ophthalmol Vis Sci 2004; 45: 2431-2437.

40 Kitaa T, Hataa Y, Aritaa R, Kawaharaa S, Miuraa M, Nakaoa $S$ et al. Role of TGF-B in proliferative vitreoretinal diseases and ROCK as a therapeutic target. Proc Natl Acad Sci USA 2008; 105: 17504-17509.

41 Yokoyama K, Kimoto K, Itoh Y. The PI3K/Akt pathway mediates the expression of type I collagen induced by TGF-2 in human retinal pigment epithelial cells. Graefes Arch Clin Exp Ophthalmol 2012; 250: 15-23.

42 Macías-Barragán J, Sandoval-Rodríguez A, Navarro-Partida J, Armendáriz-Borunda J. The multifaceted role of pirfenidone and its novel targets. Fibrogenesis Tissue Repair 2010; 3: 16.

43 Flores-Contreras L, Sandoval-Rodríguez AS, Mena-Enriquez MG, Lucano-Landeros S, Arellano-Olivera I, AlvarezÁlvarez A et al. Treatment with pirfenidone for two years decreases fibrosis, cytokine levels and enhances CB2 gene expression in patients with chronic hepatitis C. BMC Gastroenterol 2014; 14: 131.

44 Conte E, Iemmolo M, Fagone E, Gili E, Fruciano M, Genovese $\mathrm{T}$ et al. Thymosin $\beta 4$ reduces IL-17-producing cells and IL-17 expression and protects lungs from damage in bleomycin-treated mice. Immunobiology 2014; 219: 425-431.

45 Inomata M, Kamio K, Azuma A, Matsuda K, Kokuho N, Miura $\mathrm{Y}$ et al. Pirfenidone inhibits fibrocyte accumulation in the lungs in bleomycin-induced murine pulmonary fibrosis. Respir Res 2014; 15: 16.

46 Hewitson TD, Kelynack KJ, Tait MG, Martic M, Jones CL, Margolin SB. Pirfenidone reduces in vitro rat renal fibroblast activation and mitogenesis. J Nephrol 2001; 14: 453-460.

47 Jiang Y, Liu L, Pagadala J, Miller DD, Steinle JJ. Compound $49 \mathrm{~b}$ protects against blast-induced retinal injury. $J$ Neuroinflammation 2013; 10: 96.

48 Da M, Li KKW, Chan KC, Wu EX, Wong DSH. Distribution of triamcinolone acetonide after intravitreal injection into silicone oil-filled eye. Biomed Res Int 2016; 2016: 5485467.

49 Falkner CI, Binder S, Kruger A. Outcome after silicone oil removal. Br J Ophthalmol 2001; 85: 1324-1327.

50 Sun G, Lin X, Zhong H, Yang Y, Qiu X, Ye C et al. Pharmacokinetics of pirfenidone after topical administration in rabbit eye. Mol Vis 2011; 17: 2191-2196.

51 Rhee DJ, Peck RE, Belmont J, Martidis A, Liu M, Chang J et al. Intraocular pressure alterations following intravitreal triamcinolone acetonide. Br J Ophthalmol 2006; 90: 999-1003.

52 Blankenship GW. Evaluation of a single intravitreal injection of 5-fluorouracil in vitrectomy cases. Graefes Arch Clin Exp Ophthalmol 1989; 227: 565-568.

53 Zhong H, Sun G, Lin X, Wu K, Yu M. Evaluation of pirfenidone as a new postoperative antiscarring agent in experimental glaucoma surgery. Invest Ophthalmol Vis Sci 2011; 52: 3136-3142.

54 Takeda Y, Tsujino K, Kijima T, Kumanogoh A. Efficacy and safety of pirfenidone for idiopathic pulmonary fibrosis. Patient Prefer Adherence 2014; 8: 361-370.

Supplementary Information accompanies this paper on Eye website (http://www.nature.com/eye) 